\title{
Article \\ Delay Differential Equations of Fourth-Order: Oscillation and Asymptotic Properties of Solutions
}

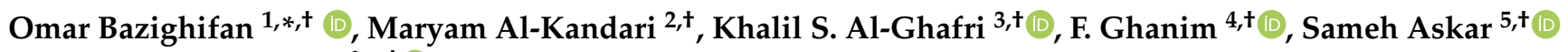 \\ and Georgia Irina Oros $6, *,+(\mathbb{D}$
}

1 Section of Mathematics, International Telematic University Uninettuno, CorsoVittorio Emanuele II, 39, 00186 Roma, Italy

2 Department of Mathematics, Kuwait University, P.O. Box 5969, Safat, Khaldiyah City 13060, Kuwait; maryam@sci.kuniv.edu.kw

3 University of Technology and Applied Sciences, P.O. Box 14, Ibri 516, Oman; khalil.ibr@cas.edu.om

4 Department of Mathematics, College of Sciences, University of Sharjah, Sharjah 27272, United Arab Emirates; fgahmed@sharjah.ac.ae

5 Department of Statistics and Operations Research, College of Science, King Saud University, P.O. Box 2455, Riyadh 11451, Saudi Arabia; saskar@ksu.edu.sa

6 Department of Mathematics and Computer Science, University of Oradea, 410087 Oradea, Romania

* Correspondence: o.bazighifan@gmail.com (O.B.); georgia_oros_ro@yahoo.co.uk (G.I.O.)

+ These authors contributed equally to this work.

Citation: Bazighifan, O.; Al-Kandari,

M.; Al-Ghafri, K.S.; Ghanim, F.; Askar,

S.; Oros, G.I. Delay Differential

Equations of Fourth-Order:

Oscillation and Asymptotic

Properties of Solutions. Symmetry

2021, 13, 2015. https://doi.org/

10.3390/sym13112015

Academic Editor: Igor Andrianov

Received: 16 September 2021

Accepted: 18 October 2021

Published: 24 October 2021

Publisher's Note: MDPI stays neutral with regard to jurisdictional claims in published maps and institutional affiliations.

Copyright: (c) 2021 by the authors. Licensee MDPI, Basel, Switzerland. This article is an open access article distributed under the terms and conditions of the Creative Commons Attribution (CC BY) license (https:/ / creativecommons.org/licenses/by/ $4.0 /)$.

\begin{abstract}
In this work, by using the comparison method and Riccati transformation, we obtain some oscillation criteria of solutions of delay differential equations of fourth-order in canonical form. These criteria complement those results in the literature. We give two examples to illustrate the main results. Symmetry plays an essential role in determining the correct methods for solutions to differential equations.
\end{abstract}

Keywords: differential equations; damped delay; fourth-order; oscillation

\section{Introduction}

Delay differential equations appear in many problems and applications especially in applications of physics, medicine, engineering, aviation and biology. Moreover, they are used in heartbeats and vibrational motion in bridges. Also, symmetrical properties contribute in Euler equation in some variational problems. In other words, it contributes to determining the appropriate method for finding the correct solution to this equation, see [1,2].

Nowadays, the oscillatory properties of differential equations has been the subject of intensive study, especially their oscillations and asymptotic, see Agarwal et al. [3] and Saker [4].

Baculikova [5], Dzurina and Jadlovska [6], and Bohner et al. [7] developed some techniques that can be used in second-order differential equations to test the qualitative and oscillatory behavior of this type of equation. Xing et al. [8] and Moaaz et al. [9] contributed to the development of the theory of oscillation by obtaining some new criteria for the oscillation of solutions of differential equations of even order. Despite the great interest by many researchers to obtain qualitative and oscillatory properties of different types of equations such as fractional order differential equations, the oscillation criteria for delay differential equations have received some few studies, although such equations are of usefulness and importance in some fields of science for its appearance in many applications. Many researchers have discussed the qualitative and oscillatory behavior of differential equations with neutral and damped terms, see [10-21]. 
For even-order differential equations, Park et al. [22] were interested in studying the oscillation conditions of the equations

$$
\left(\alpha(\imath)\left(y^{(n-1)}(\imath)\right)^{\kappa}\right)^{\prime}+\vartheta(\imath) y^{\ell}(\phi(\imath))=0,
$$

(or some of its special cases) where $\kappa$ and $\ell$ are ratios of odd positive integers, $\ell \leq \kappa$ and they only focused on studying the oscillation of (1) in the canonical case, that is,

$$
\int_{l_{0}}^{\infty} \alpha^{-1 / \kappa}(s) \mathrm{d} s=\infty .
$$

In [23], Zhang et al. examined the qualitative properties of (1) in the noncanonical case, that is,

$$
\int_{l_{0}}^{\infty} \alpha^{-1 / \kappa}(s) \mathrm{d} s<\infty .
$$

Baculikova et al. [24] presented oscillation results for Emden-Fowler equation

$$
\left[\alpha(\imath)\left(y^{(n-1)}(\imath)\right)^{\kappa}\right]^{\prime}+\vartheta(\imath) f(y(\phi(\imath)))=0
$$

and used the Riccati method to obtain some oscillation theorems. Moreover, by introducing a generalized Riccati substitution, Moaaz and Muhib [25] extended the technique used in [26] to study the oscillation of (1).

Zhang et al. [27] discussed some oscillation theorems for (3) where $\kappa=\ell$ and contributed to improving the oscillatory properties for this equation.

In case $n=4$, Zhang et al. [28] investigated some oscillation theorems of equation

$$
\left[\alpha(\imath)\left(y^{\prime \prime \prime}(\imath)\right)^{\kappa}\right]^{\prime}+\vartheta(\imath) f(y(\phi(\imath)))=0,
$$

where $\kappa$ and $\ell$ are the ratio of odd natural numbers.

Bazighifan [29] investigated the oscillation of equation

$$
\left[\alpha(\imath)\left(y^{\prime \prime \prime}(\imath)\right)^{\kappa}\right]^{\prime}+\vartheta(\imath) y^{\ell}(\phi(\imath))=0 .
$$

The authors in [30] considered that Equation (4) where $\kappa=\ell=1$ is oscillatory if

$$
\int_{\iota_{0}}^{\infty}\left(\zeta(s) \vartheta(s) \frac{r}{2} \phi^{2}(s)-\frac{1}{4 \zeta(s) \alpha(s)}\left[\frac{s^{\prime}(s)}{\varsigma(s)}-\frac{\beta(s)}{\alpha(s)}\right]^{2}\right) d s=\infty,
$$

for some $r \in(0,1)$, and

$$
\int_{l_{0}}^{\infty}\left[\theta(s) \int_{s}^{\infty}\left[\frac{1}{\alpha(v)} \int_{v}^{\infty} \vartheta(s)\left(\frac{\phi^{2}(s)}{s^{2}}\right) d s\right] d v-\frac{\left(\theta^{\prime}(s)\right)^{2}}{4 \theta(s)}\right] d s=\infty
$$

and under the condition (6).

Based on the above results of previous scholars, in this work, we are concerned with the following differential equations with delay term of the form

$$
\left(\alpha(\imath)\left(y^{\prime \prime \prime}(\imath)\right)^{\kappa}\right)^{\prime}+\sum_{j=1}^{m} \vartheta_{j}(\imath) y^{\ell}\left(\phi_{j}(\imath)\right)=0
$$

and

$$
\left(\alpha(\imath)\left(y^{\prime \prime \prime}(\imath)\right)^{\kappa}\right)^{\prime}+\beta(\imath)\left(y^{\prime \prime \prime}(\imath)\right)^{\kappa}+\sum_{j=1}^{m} \vartheta_{j}(\imath) y^{\ell}\left(\phi_{j}(\imath)\right)=0 .
$$


where $\kappa$ and $\ell$ are quotient of odd positive integers and under the conditions:

Hypothesis $\left(\mathbf{H}_{\mathbf{1}}\right) . \alpha, \beta, \vartheta \in C\left(\left[\imath_{0}, \infty\right),[0, \infty)\right), \phi_{j}(\imath) \in C\left(\left[\imath_{0}, \infty\right), \mathbb{R}\right)$,

Hypothesis $\left(\mathbf{H}_{2}\right) . \alpha^{\prime}(\imath)+\beta(\imath) \geq 0, \alpha(\imath)>0, \vartheta_{j}(\imath)>0, \phi_{j}(\imath) \leq \imath, \lim _{l \rightarrow \infty} \phi_{j}(\imath)=\infty, j=$ $1,2, \ldots, r$. Throughout this article, we study (3) under the hypothesis

$$
\int_{l_{0}}^{\infty} \frac{1}{\alpha^{1 / \kappa}(s)} \mathrm{d} s=\infty
$$

and (4) under the condition

$$
\int_{l_{0}}^{\infty}\left[\frac{1}{\alpha(s)} \exp \left(-\int_{\imath_{0}}^{s} \frac{\beta(w)}{\alpha(w)} d w\right)\right]^{1 / \kappa} d s=\infty
$$

Definition 1. A nontrivial solution y of (3) and (4) is called oscillatory or nonoscillatory according if it contains does or does not have infinitely many zeros.

Definition 2. Equations (3) and (4) are called oscillatory if each of their solutions is oscillatory.

The motivation for this article is to continue the previous works [23,30], which discussed the oscillatory properties of equations in a canonical form.

The authors in $[23,30]$ used the comparison method that differs from the one we used in this work. So, the technique used gives more accurate criteria. Moreover, these criteria complement those results in the literature.

The main idea of our method in this article is to make a comparison with a first-order differential equation whose oscillatory behavior has been known before, also we use the Riccati transformation to reduce the order of the studied equation. Thus, we claim that the obtained results are new and complement those results in the literature.

To obtain our results, we shall need the following lemmas:

Lemma 1 ([31]). If $y^{(m)}(\imath)>0, m=0,1, \ldots, r$, and $y^{(r+1)}(\imath)<0$, then

$$
\frac{y(\imath)}{\imath^{r} / r !} \geq \frac{y^{\prime}(\imath)}{\imath^{r-1} /(r-1) !} .
$$

Lemma 2 ([32]). Let $w \in C^{r}\left(\left[\imath_{0}, \infty\right),(0, \infty)\right)$ and $w^{(r)}(\imath)$ is of a fixed sign, on $\left[\iota_{0}, \infty\right)$ such that, for all $\imath \geq \imath_{1}$,

$$
w^{(r-1)}(\imath) w^{(r)}(\imath) \leq 0 .
$$

If we have $\lim _{\imath \rightarrow \infty} w(\imath) \neq 0$, then there exists $\imath_{\lambda} \geq \imath_{0}$ such that

$$
w(\imath) \geq \frac{\lambda}{(r-1) !} \imath^{r-1}\left|w^{(r-1)}(\imath)\right|
$$

for every $\lambda \in(0,1)$ and $\iota \geq \imath_{\lambda}$.

Lemma 3 ([33]). Let $F>0$. Then

$$
E u-F u^{(v+1) / v} \leq \frac{v^{v}}{(v+1)^{v+1}} E^{v+1} F^{-v} .
$$

For convenience, we denote:

$$
R(\imath):=\int_{\imath}^{\infty}\left(\frac{1}{\alpha(x)} \int_{x}^{\infty} \sum_{j=1}^{m} \vartheta_{j}(s) \mathrm{d} s\right)^{1 / \kappa} \mathrm{d} x,
$$




$$
\begin{gathered}
\widetilde{R}(\imath):=\mu_{2}^{\ell / \kappa} \int_{\imath}^{\infty}\left(\frac{1}{\alpha(x)} \int_{x}^{\infty} \sum_{j=1}^{m} \vartheta_{j}(s)\left(\frac{\phi_{j}(s)}{s}\right)^{\ell} \mathrm{d} s\right)^{1 / \kappa} \mathrm{d} x, \\
\zeta_{\imath_{0}}(\imath):=\exp \left(\int_{\imath_{0}}^{\imath} \frac{\beta(x)}{\alpha(x)} \mathrm{d} x\right)
\end{gathered}
$$

and

$$
\widehat{R}(\imath):=\mu_{2}^{\ell / \kappa} \int_{\imath}^{\infty}\left(\frac{1}{\alpha(x) \zeta_{\iota_{0}}(\imath)} \int_{x}^{\infty} \zeta_{\iota_{0}}(\imath) \sum_{j=1}^{m} \vartheta_{j}(s)\left(\frac{\phi_{j}(s)}{s}\right)^{\ell} \mathrm{d} s\right)^{1 / \kappa} \mathrm{d} x,
$$

where $\mu_{2} \in(0,1)$.

\section{Oscillation Criteria for (3)}

Lemma 4. Let (5) holds and $y$ is an eventually positive solution of (3), then $y^{\prime}>0$ and $y^{\prime \prime \prime}>0$.

Proof. The proof is clear and easy and thus it has been deleted.

Theorem 1. If

$$
x^{\prime}(\imath)+\frac{\lambda^{\ell}}{6^{\ell}} \frac{\sum_{j=1}^{m} \vartheta_{j}(\imath) \phi_{j}^{3 \ell}(\imath)}{\alpha^{\ell / \kappa}\left(\phi_{j}(\imath)\right)} x^{\ell / \kappa}\left(\phi_{j}(\imath)\right)=0, \lambda \in(0,1),
$$

is oscillatory, then (3) is oscillatory.

Proof. Let (3) has a nonoscillatory solution in $\left[\imath_{0}, \infty\right)$. Then $y(\imath)>0$ and $y\left(\phi_{j}(\imath)\right)>0$ for $\imath \geq \imath_{1}$. Let

$$
x(\imath):=\alpha(\imath)\left(y^{\prime \prime \prime}(\imath)\right)^{\kappa}>0 \text { [from Lemma 4], }
$$

which with (3) gives

$$
x^{\prime}(\imath)+\sum_{j=1}^{m} \vartheta_{j}(\imath) y^{\ell}\left(\phi_{j}(\imath)\right)=0 .
$$

Since $\lim _{\imath \rightarrow \infty} y(\imath) \neq 0$. Thus, by Lemma 2, we obtain

$$
y^{\ell}\left(\phi_{j}(\imath)\right) \geq \frac{\lambda^{\ell}}{6^{\ell}} \phi_{j}^{3 \ell}(\imath)\left(y^{\prime \prime \prime}\left(\phi_{j}(\imath)\right)\right)^{\ell},
$$

for all $\lambda \in(0,1)$. By (9) and (10), we see that

$$
x^{\prime}(\imath)+\frac{\lambda^{\ell}}{6^{\ell}} \sum_{j=1}^{m} \vartheta_{j}(\imath) \phi_{j}^{3 \ell}(\imath)\left(y^{\prime \prime \prime}\left(\phi_{j}(\imath)\right)\right)^{\ell} \leq 0 .
$$

So, we obtain $x(\imath)>0$ and

$$
x^{\prime}(\imath)+\frac{\lambda^{\ell}}{6^{\ell}} \frac{\sum_{j=1}^{m} \vartheta_{j}(\imath) \phi_{j}^{3 \ell}(\imath)}{\alpha^{\ell / \kappa}\left(\phi_{j}(\imath)\right)} x^{\ell / \kappa}\left(\phi_{j}(\imath)\right) \leq 0 .
$$

When using ([13], Theorem 1), we notice that (8) is nonoscillatory, which is an obvious contradiction, so the proof of this theorem is complete.

Corollary 1. Let $\kappa=\ell$ and

$$
\liminf _{\imath \rightarrow \infty} \int_{\phi(l)}^{\imath} \frac{\lambda^{\ell}}{6^{\ell}} \frac{\sum_{j=1}^{m} \vartheta_{j}(s) \phi_{j}^{3 \ell}(s)}{\alpha^{\ell / \kappa}\left(\phi_{j}(s)\right)} \mathrm{d} s>\frac{1}{\mathrm{e}^{\prime}},
$$


then (3) is oscillatory.

Lemma 5. If

$$
\int_{\imath_{0}}^{\infty}\left(M^{\ell-\kappa} \zeta(\imath) \sum_{j=1}^{m} \vartheta_{j}(\imath) \frac{\phi_{j}^{3 \kappa}(\imath)}{\imath^{3 \kappa}}-\frac{2^{\kappa}}{(\kappa+1)^{\kappa+1}} \frac{\alpha(\imath)\left(s^{\prime}(\imath)\right)^{\kappa+1}}{\mu^{\kappa} \imath^{2 \kappa} S^{\kappa}(\imath)}\right) \mathrm{d} s=\infty,
$$

for some $\mu \in(0,1)$, then $y^{\prime \prime}<0$.

Proof. If $y^{\prime \prime}(\imath)>0$. When using Lemmas 1 and 2 , we obtain

$$
\frac{y\left(\phi_{j}(\imath)\right)}{y(\imath)} \geq \frac{\phi_{j}^{3}(\imath)}{\imath^{3}}
$$

and

$$
y^{\prime}(\imath) \geq \frac{\mu}{2} \imath^{2} y^{\prime \prime \prime}(\imath)
$$

Let

$$
\psi(\imath):=\varsigma(\imath) \frac{\alpha(\imath)\left(y^{\prime \prime \prime}(\imath)\right)^{\kappa}}{y^{\kappa}(\imath)}>0 .
$$

From (13)-(15), we find

$$
\psi^{\prime}(\imath) \leq \frac{\varsigma^{\prime}(\imath)}{\varsigma(\imath)} \eta(\imath)-\varsigma(\imath) \sum_{j=1}^{m} \vartheta_{j}(\imath) \frac{\phi_{j}^{3 \kappa}(\imath)}{\imath^{3 \kappa}} y^{\ell-\kappa}\left(\phi_{j}(\imath)\right)-\frac{\kappa \mu}{2} \frac{\imath^{2}}{\varsigma^{1 / \kappa}(\imath) \alpha^{1 / \kappa}(\imath)} \psi^{1+1 / \kappa}(\imath)
$$

Since $y^{\prime}(\imath)>0$. From Lemmas 3 with $E=\varsigma^{\prime} / \varsigma, F=\kappa \mu \imath^{2} /\left(2 \alpha^{1 / \kappa}(\imath) \varsigma^{1 / \kappa}(\imath)\right)$ and $u=\psi$, we see that

$$
\psi^{\prime}(\imath) \leq-M^{\ell-\kappa} \varsigma(\imath) \sum_{j=1}^{m} \vartheta_{j}(\imath) \frac{\phi_{j}^{3 \kappa}(\imath)}{\imath^{3 \kappa}}+\frac{2^{\kappa}}{(\kappa+1)^{\kappa+1}} \frac{\alpha(\imath)\left(\zeta^{\prime}(\imath)\right)^{\kappa+1}}{\mu^{\kappa} \imath^{2 \kappa} \zeta^{\kappa}(\imath)} .
$$

This implies that

$$
\int_{\imath_{1}}^{\imath}\left(M^{\ell-\kappa} \zeta(\imath) \sum_{j=1}^{m} \vartheta_{j}(\imath) \frac{\phi_{j}^{3 \kappa}(\imath)}{\imath^{3 \kappa}}-\frac{2^{\kappa}}{(\kappa+1)^{\kappa+1}} \frac{\alpha(\imath)\left(\zeta^{\prime}(\imath)\right)^{\kappa+1}}{\mu^{\kappa} \imath^{2 \kappa} \varsigma^{\kappa}(\imath)}\right) \mathrm{d} s \leq \psi\left(\imath_{1}\right),
$$

which contradicts (12). The proof is complete.

Theorem 2. If

$$
u^{\prime \prime}(\imath)+M^{\ell-\kappa} \widetilde{R}(\imath) u(\imath)=0
$$

is oscillatory, then (3) is oscillatory.

Proof. Proceeding as in the proof of Theorem 1. By Lemmas 2 and 4, we have

$$
y^{\prime}(\imath)>0, y^{\prime \prime}(\imath)<0 \text { and } y^{\prime \prime \prime}(l)>0 \text {. }
$$

Now, integrating (3) from $\imath$ to $b$, we have

$$
\alpha(b)\left(y^{\prime \prime \prime}(b)\right)^{\kappa}=\alpha(\imath)\left(y^{\prime \prime \prime}(\imath)\right)^{\kappa}-\int_{\imath}^{b} \sum_{j=1}^{m} \vartheta_{j}(s) y^{\ell}\left(\phi_{j}(s)\right) \mathrm{d} s .
$$


By Lemma 3 in [33] with (18), we get

$$
\frac{y\left(\phi_{j}(\imath)\right)}{y(\imath)} \geq \lambda \frac{\phi_{j}(\imath)}{\imath}
$$

which with (19) gives

$$
\alpha(b)\left(y^{\prime \prime \prime}(b)\right)^{\kappa}-\alpha(\imath)\left(y^{\prime \prime \prime}(\imath)\right)^{\kappa}+\lambda^{\ell} \int_{\imath}^{b} \sum_{j=1}^{m} \vartheta_{j}(s)\left(\frac{\phi_{j}(s)}{s}\right)^{\ell} y^{\ell}(s) \mathrm{d} s \leq 0 .
$$

By $y^{\prime}>0$, we find

$$
\alpha(b)\left(y^{\prime \prime \prime}(b)\right)^{\kappa}-\alpha(\imath)\left(y^{\prime \prime \prime}(\imath)\right)^{\kappa}+\lambda^{\ell} y^{\ell}(\imath) \int_{\imath}^{b} \sum_{j=1}^{m} \vartheta_{j}(s)\left(\frac{\phi_{j}(s)}{s}\right)^{\ell} \mathrm{d} s \leq 0 .
$$

Taking $b \rightarrow \infty$, we obtain

$$
-\alpha(\imath)\left(y^{\prime \prime \prime}(\imath)\right)^{\kappa}+\lambda^{\ell} y^{\ell}(\imath) \int_{\imath}^{\infty} \sum_{j=1}^{m} \vartheta_{j}(s)\left(\frac{\phi_{j}(s)}{s}\right)^{\ell} \mathrm{d} s \leq 0,
$$

that is

$$
y^{\prime \prime \prime}(\imath) \geq \frac{\lambda^{\ell / \kappa}}{\alpha^{1 / \kappa}(\imath)} y^{\ell / \kappa}(\imath)\left(\int_{\imath}^{\infty} \sum_{j=1}^{m} \vartheta_{j}(s)\left(\frac{\phi_{j}(s)}{s}\right)^{\ell} \mathrm{d} s\right)^{1 / \kappa} .
$$

Integrating from $\imath$ to $\infty$, we get

$$
-y^{\prime \prime}(\imath) \geq \lambda^{\ell / \kappa} y^{\ell / \kappa}(\imath) \int_{\imath}^{\infty}\left(\frac{1}{\alpha(x)} \int_{x}^{\infty} \sum_{j=1}^{m} \vartheta_{j}(s)\left(\frac{\phi_{j}(s)}{s}\right)^{\ell} \mathrm{d} s\right)^{1 / \kappa} \mathrm{d} x,
$$

hence

$$
y^{\prime \prime}(\imath) \leq-\widetilde{R}(\imath) y^{\ell / \kappa}(\imath)
$$

Now, if we define $\eta$ by

$$
\eta(\imath)=\frac{y^{\prime}(\imath)}{y(\imath)}
$$

then $\eta(\imath)>0$ for $\imath \geq \imath_{1}$, and

$$
\eta^{\prime}(\imath)=\frac{y^{\prime \prime}(\imath)}{y(\imath)}-\left(\frac{y^{\prime}(\imath)}{y(\imath)}\right)^{2}
$$

From (21), we find

$$
\eta^{\prime}(\imath) \leq-\widetilde{R}(\imath) \frac{y^{\ell / \kappa}(\imath)}{y(\imath)}-\eta^{2}(\imath) .
$$

Since $y^{\prime}(\imath)>0$. Thus, (22) becomes

$$
\eta^{\prime}(\imath)+\eta^{2}(\imath)+M^{\ell-\kappa} \widetilde{R}(\imath) \leq 0,
$$

From [17], we obtain (17) is nonoscillatory, which contradicts, so the proof of this theorem is complete.

Theorem 3. If $\ell \geq \kappa$ and

$$
\left(\frac{1}{\phi_{j}^{\prime}(\imath)} u^{\prime}(\imath)\right)^{\prime}+M^{\ell / \kappa-1} R(\imath) u(\imath)=0
$$


is oscillatory, then (3) is oscillatory.

Proof. Let (12) and (19) hold. So, we note from $\phi_{j}^{\prime}(\imath) \geq 0$ and $y^{\prime}(\imath) \geq 0$

$$
\alpha(b)\left(y^{\prime \prime \prime}(b)\right)^{\kappa}-\alpha(\imath)\left(y^{\prime \prime \prime}(\imath)\right)^{\kappa}+y^{\ell}\left(\phi_{j}(\imath)\right) \int_{\imath}^{b} \sum_{j=1}^{m} \vartheta_{j}(s) \mathrm{d} s \leq 0 .
$$

Thus, (18) becomes

$$
y^{\prime \prime}(\imath) \leq-R(\imath) y^{\ell / \kappa}\left(\phi_{j}(\imath)\right)
$$

Let

$$
\omega(\imath)=\frac{y^{\prime}(\imath)}{y\left(\phi_{j}(\imath)\right)}
$$

then $\omega(\imath)>0$ for $\imath \geq \imath_{1}$, and

$$
\begin{aligned}
\omega^{\prime}(\imath) & =\frac{y^{\prime \prime}(\imath)}{y\left(\phi_{j}(\imath)\right)}-\frac{y^{\prime}(\imath)}{y^{2}\left(\phi_{j}(\imath)\right)} y^{\prime}\left(\phi_{j}(\imath)\right) \phi_{j}^{\prime}(\imath) \\
& \leq \frac{y^{\prime \prime}(\imath)}{y\left(\phi_{j}(\imath)\right)}-\phi_{j}^{\prime}(\imath)\left(\frac{y^{\prime}(l)}{y\left(\phi_{j}(l)\right)}\right)^{2}
\end{aligned}
$$

From (26) and (27), we find

$$
\omega^{\prime}(\imath)+M^{\ell / \kappa-1} R(\imath)+\phi_{j}^{\prime}(\imath) \omega^{2}(\imath) \leq 0 .
$$

From [17], we find (24) is nonoscillatory, which is a contradiction, thus the proof of the theorem is completed.

Corollary 2. If $\ell=\kappa$ and

$$
\lim _{\imath \rightarrow \infty} \frac{1}{G\left(\imath, \imath_{0}\right)} \int_{\imath_{0}}^{\imath}\left(G(\imath, s) \widetilde{R}(s)-\frac{1}{4} h^{2}(\imath, s)\right) \mathrm{d} s=\infty
$$

or

$$
\liminf _{l \rightarrow \infty} \int_{l}^{\infty} \widetilde{R}(s) \mathrm{d} s>\frac{1}{4}
$$

then (3) is oscillatory.

Corollary 3. Let $\ell=\kappa$ and (12) hold. If

$$
\imath^{2} \widetilde{R}(s) \geq \varepsilon
$$

and

$$
\limsup _{\imath \rightarrow \infty}\left(\imath^{\varepsilon-1} \int_{l_{0}}^{\imath} s^{2-\varepsilon} \widetilde{R}(s) \mathrm{d} s+\imath^{1-\widetilde{\varepsilon}} \int_{\imath}^{\infty} s^{\widetilde{\varepsilon}} \widetilde{R}(s) \mathrm{d} s\right)>1,
$$

where $\widetilde{\varepsilon}=\frac{1}{2}(1-\sqrt{1-4 \varepsilon})$ and $\varepsilon \in(0,1 / 4]$, then (3) is oscillatory. 


\section{Oscillation Results for Equation (4)} we find

In this section, we shall get oscillation conditions for (4) by converting to (3), easily,

$$
\begin{aligned}
\frac{1}{\zeta_{l_{0}}(\imath)} \frac{\mathrm{d}}{\mathrm{d} \imath}\left(\mu(\imath) \alpha(\imath)\left(y^{\prime \prime \prime}(\imath)\right)^{\kappa}\right) & =\frac{1}{\zeta_{l_{0}}(\imath)}\left[\zeta_{\imath_{0}}(\imath)\left(\alpha(\imath)\left(y^{\prime \prime \prime}(\imath)\right)^{\kappa}\right)^{\prime}+\zeta_{\imath_{0}}^{\prime}(\imath) \alpha(\imath)\left(y^{\prime \prime \prime}(\imath)\right)^{\kappa}\right] \\
& =\left(\alpha(\imath)\left(y^{\prime \prime \prime}(\imath)\right)^{\kappa}\right)^{\prime}+\frac{\zeta_{l_{0}}^{\prime}(\imath)}{\zeta_{\imath_{0}}(\imath)} \alpha(\imath)\left(y^{\prime \prime \prime}(\imath)\right)^{\kappa}, \\
& =\left(\alpha(\imath)\left(y^{\prime \prime \prime}(\imath)\right)^{\kappa}\right)^{\prime}+\beta(\imath)\left(y^{\prime \prime \prime}(\imath)\right)^{\kappa},
\end{aligned}
$$

which with (4) gives

$$
\left(\zeta_{\imath_{0}}(\imath) \alpha(\imath)\left(y^{\prime \prime \prime}(\imath)\right)^{\kappa}\right)^{\prime}+\zeta_{\imath_{0}}(\imath) \sum_{j=1}^{m} \vartheta_{j}(\imath) y^{\ell}\left(\phi_{j}(\imath)\right)=0 .
$$

Corollary 4. Assume that $\kappa=\ell, \lambda \in(0,1)$, (6) holds. If

$$
\liminf _{\imath \rightarrow \infty} \int_{\phi(\imath)}^{\imath} \frac{\lambda^{\ell}}{6^{\ell}} \frac{\zeta_{l_{0}}(s) \sum_{j=1}^{m} \vartheta_{j}(s) \phi_{j}^{3 \ell}(s)}{\zeta_{l_{0}}^{\ell / \kappa}\left(\phi_{j}(s)\right) \alpha^{\ell / \kappa}\left(\phi_{j}(s)\right)} \mathrm{d} s>\frac{1}{\mathrm{e}^{\prime}}
$$

then (4) is oscillatory.

Corollary 5. Let $\ell=\kappa,(6)$ and

$$
\int_{\imath_{0}}^{\infty}\left(M^{\ell-\kappa} \varsigma(\imath) \zeta_{l_{0}}(\imath) \sum_{j=1}^{m} \vartheta_{j}(\imath) \frac{\phi_{j}^{3 \kappa}(\imath)}{\imath^{3 \kappa}}-\frac{2^{\kappa}}{(\kappa+1)^{\kappa+1}} \frac{\alpha(\imath) \zeta_{l_{0}}(\imath)\left(\varsigma^{\prime}(\imath)\right)^{\kappa+1}}{\mu^{\kappa} \imath^{2 \kappa} \varsigma^{\kappa}(\imath)}\right) \mathrm{d} s=\infty .
$$

If

$$
\lim _{l \rightarrow \infty} \frac{1}{G\left(\imath, \imath_{0}\right)} \int_{\imath_{0}}^{\imath}\left(G(\imath, x) \widehat{R}(x)-\frac{1}{4} h^{2}(\imath, x)\right) \mathrm{d} x=\infty
$$

or

$$
\liminf _{l \rightarrow \infty} \int_{\imath}^{\infty} \widehat{R}(x) \mathrm{d} x>\frac{1}{4},
$$

then (4) is oscillatory.

Corollary 6. Let $\ell=\kappa$ and (30) hold. If

$$
\imath^{2} \widehat{R}(s) \geq \varepsilon
$$

and

$$
\limsup _{l \rightarrow \infty}\left(\imath^{\varepsilon-1} \int_{\imath_{0}}^{\imath} s^{2-\varepsilon} \widehat{R}(s) \mathrm{d} s+\imath^{1-\widetilde{\varepsilon}} \int_{\imath}^{\infty} s^{\widetilde{\varepsilon}} \widehat{R}(s) \mathrm{d} s\right)>1,
$$

then (4) is oscillatory.

Example 1. Let the equation:

$$
\left(\imath^{3}\left(y^{\prime \prime \prime}(\imath)\right)^{3}\right)^{\prime}+\frac{\vartheta_{0}}{\imath^{7}} y^{3}(\gamma \imath)=0,
$$

where $\imath \geq 1, \gamma \in(0,1]$ and $\vartheta_{0}>0$. We note that $\kappa=\ell=3, \phi_{j}(\imath)=\gamma \imath, \alpha(\imath)=\imath^{3}$, and $\vartheta(\imath)=\vartheta_{0} / \imath^{7}$. So, we obtain

$$
\widetilde{R}(\imath)=\lambda\left(\frac{\vartheta_{0}}{6}\right)^{1 / 3} \gamma \frac{1}{2 \imath^{2}}
$$


By Corollarys 1 and 2, we find that Equation (31) is oscillatory if

$$
\begin{gathered}
\vartheta_{0}>\frac{6^{3}}{e\left(\ln \frac{1}{\gamma}\right) \gamma^{6}} \\
\vartheta_{0}>\left(\frac{3^{4}}{2}\right) \frac{1}{\gamma^{9}}
\end{gathered}
$$

and

$$
\vartheta_{0}>6\left(\frac{1}{4 \gamma}\right)^{3}
$$

So, Equation (31) is oscillatory if

$$
\vartheta_{0}>\max \left\{\left(\frac{3^{4}}{2}\right) \frac{1}{\gamma^{9}}, 6\left(\frac{1}{4 \gamma}\right)^{3}\right\}=\left(\frac{3^{4}}{2}\right) \frac{1}{\gamma^{9}} .
$$

Example 2. Let the equation

$$
y^{(4)}(\imath)+\frac{1}{\imath} y^{(3)}(\imath)+\frac{\theta_{0}}{\imath^{4}} y\left(\frac{\imath}{2}\right)=0,
$$

where $\imath \geq 1$, and $\theta_{0}>0$ is a constant. Let $\kappa=\ell=1, n=4, \alpha(\imath)=1, \phi_{j}(\imath)=\imath / 2, \beta(\imath)=1 / \imath$ and $\vartheta(\imath)=\theta_{0} / \imath^{4}$. Then

$$
\zeta_{\imath_{0}}(\imath)=\imath, \zeta_{\imath_{0}}\left(\phi_{j}(\imath)\right)=\imath / 2
$$

So, we see that

$$
\begin{aligned}
& \liminf _{\imath \rightarrow \infty} \int_{\phi(\imath)}^{\imath} \frac{\lambda^{\ell}}{\sigma^{\ell}} \frac{\zeta_{\imath_{0}}(s) \sum_{j=1}^{m} \vartheta_{j}(s) \phi_{j}^{3 \ell}(s)}{\zeta_{\imath_{0}}^{\ell / \kappa}\left(\phi_{j}(s)\right) \alpha^{\ell / \kappa}\left(\phi_{j}(s)\right)} \mathrm{d} s \\
= & \liminf _{\imath \rightarrow \infty} \int_{\imath / 2}^{\imath} \frac{\lambda}{3} \frac{\theta_{0}}{\imath^{4}}\left(\frac{\imath^{3}}{8}\right) \mathrm{d} s=\frac{\lambda \theta_{0}}{24} \ln 2 .
\end{aligned}
$$

The condition become

$$
\theta_{0}>\frac{24}{\lambda \mathrm{e} \ln 2}
$$

Using Corollary 4 , all solution of (33) is oscillatory if $\theta_{0}>\frac{24}{\lambda \mathrm{e} \ln 2}$ for all $\lambda \in(0,1)$.

\section{Conclusions}

In this work, a large amount of attention has been focused on the oscillation problem of Equations (3) and (4). By Riccati transformation and comparison technique, we establish some new oscillatory properties. These criteria complement those results in the literature. For future consideration, it will be of a great importance to study the qualitative properties of $p$-Laplacian differential equations

$$
\left[\alpha(\imath)\left|\left(y^{(n-1)}(\imath)\right)\right|^{p-2} y^{(n-1)}(\imath)\right]^{\prime}+\sum_{j=1}^{m} \vartheta_{j}(\imath) y^{\ell}\left(\phi_{j}(\imath)\right)=0,
$$

under the assumption that

$$
\int_{\imath_{0}}^{\infty} \frac{1}{\alpha^{1 / p-1}(s)} \mathrm{d} s<\infty
$$

where $p>1$ is a constant. 
Author Contributions: CConceptualization, O.B., M.A.-K., K.S.A.-G., F.G., S.A. and G.I.O.; Data curation, O.B., M.A.-K., K.S.A.-G., F.G., S.A. and G.I.O.; Formal analysis, O.B., M.A.-K., K.S.A.-G., F.G., S.A. and G.I.O.; Investigation, O.B., M.A.-K., K.S.A.-G., F.G., S.A. and G.I.O.; Methodology, O.B., M.A.-K., K.S.A.-G., F.G., S.A. and G.I.O. All authors read and agreed to the published version of the manuscript.

Funding: This research received no external funding.

Institutional Review Board Statement: Not applicable.

Informed Consent Statement: Not applicable.

Data Availability Statement: Not applicable.

Acknowledgments: Research Supporting Project number (RSP-2021/167), King Saud University, Riyadh, Saudi Arabia.

Conflicts of Interest: The author declare no conflict of interest.

\section{References}

1. Hale, J.K. Theory of Functional Differential Equations; Springer: New York, NY, USA, 1977.

2. Agarwal, R.P.; Bohner, M.; Li, T.; Zhang, C. A new approach in the study of oscillatory behavior of even-order neutral delay diferential equations. Appl. Math. Comput. 2013, 225, 787-794.

3. Agarwal, R.; Grace, S.; O'Regan, D. Oscillation Theory for Difference and Functional Differential Equations; Kluwer Academic Publisher: Dordrecht, The Netherland, 2000.

4. Saker, S. Oscillation Theory of Delay Differential and Difference Equations: Second and Third Orders; LAP Lambert Academic Publishing: Saarbrucken, Germany, 2010.

5. Baculikova, B. Oscillation of second-order nonlinear noncanonical differential equations with deviating argument. Appl. Math. Lett. 2019, 91, 68-75. [CrossRef]

6. Dzrina, J.; Jadlovska, I. A note on oscillation of second-order delay differential equations. Appl. Math. Lett. 2017, 69, 126-132. [CrossRef]

7. Bohner, M.; Grace, S.R.; Jadlovska, I. Sharp oscillation criteria for second-order neutral delay differential equations. Math. Meth. Appl. Sci. 2020, 43, 10041-10053. [CrossRef]

8. Xing, G.; Li, T.; Zhang, C. Oscillation of higher-order quasi linear neutral differential equations. Adv. Differ. Equ. 2011, 2011, 1-10. [CrossRef]

9. Moaaz, O.; Awrejcewicz, J.; Bazighifan, O. A New Approach in the Study of Oscillation Criteria of Even-Order Neutral Differential Equations. Mathematics 2020, 8, 197. [CrossRef]

10. Kiguradze, I.T.; Chanturiya, T.A. Asymptotic Properties of Solutions of Nonautonomous Ordinary Differential Equations; Kluwer Academic Publisher: Dordrecht, The Netherland, 1993.

11. Agarwal, R.P.; Bazighifan, O.; Ragusa, M.A.-K. Nonlinear Neutral Delay Differential Equations of Fourth-Order: Oscillation of Solutions. Entropy 2021, 23, 129. [CrossRef]

12. Tang, S.; Li, T.; Thandapani, E. Oscillation of higher-order half-linear neutral differential equations. Demonstr. Math. 2013, 1, 101-109. [CrossRef]

13. Philos, C.G. On the existence of nonoscillatory solutions tending to zero at $\infty$ for differential equations with positive delays. Arch. Math. 1981, 36, 168-178. [CrossRef]

14. Chatzarakis, G.E.; Li, T. Oscillations of differential equations generated by several deviating arguments. Adv. Differ. Equ. 2017, 2017, 1-24. [CrossRef]

15. Chatzarakis, G.E.; Li, T. Oscillation criteria for delay and advanced differential equations with nonmonotone arguments. Complexity 2018, 2018, 8237634. [CrossRef]

16. Ghanim, F.; Al-Janaby, H.F.; Bazighifan, O. Some New Extensions on Fractional Differential and Integral Properties for MittagLeffler Confluent Hypergeometric Function. Fractal Fract. 2021, 5, 143. [CrossRef]

17. Agarwal, R.; Shieh, S.L.; Yeh, C.C. Oscillation criteria for second order retarde ddifferential equations. Math. Comput. Model. 1997, 26, 1-11. [CrossRef]

18. Bazighifan, O.; Ghanim, F.; Awrejcewicz, J.; Al-Ghafri, K.S.; Al-Kandari, M. New Criteria for Oscillation of Half-Linear Differential Equations with $p$-Laplacian-Like Operators. Mathematics 2021, 9, 2584. [CrossRef]

19. Hille, E. Non-oscillation theorems. Trans. Am. Math. Soc. 1948, 64, 234-253. [CrossRef]

20. Philos, C.G. Oscillation theorems for linear differential equation of second order. Arch. Math. 1989, 53, 483-492. [CrossRef]

21. Zhang, Q.; Yan, J. Oscillation behavior of even order neutral differential equations with variable coefficients. Appl. Math. Lett. 2006, 19, 1202-1206. [CrossRef]

22. Park, C.; Moaaz, O.; Bazighifan, O. Oscillation results for higher order differential equations. Axioms 2020, 9, 14. [CrossRef]

23. Zhang, C.; Li, T.; Suna, B.; Thandapani, E. On the oscillation of higher-order half-linear delay differential equations. Appl. Math. Lett. 2011, 24, 1618-1621. [CrossRef] 
24. Baculikova, B.; Dzurina, J.; Graef, J.R. On the oscillation of higher-order delay differential equations. Math. Slovaca 2012, 187, 387-400. [CrossRef]

25. Moaaz, O.; Muhib, A. New oscillation criteria for nonlinear delay differential equations of fourth-order. Appl. Math. Comput. 2020, 377, 125192. [CrossRef]

26. Agarwal, R.P.; Zhang, C.; Li, T. Some remarks on oscillation of second order neutral differential equations. Appl. Math. Comput. 2016, 274, 178-181. [CrossRef]

27. Zhang, C.; Agarwal, R.P.; Bohner, M.; Li, T. New results for oscillatory behavior of even-order half-linear delay differential equations. Appl. Math. Lett. 2013, 26, 179-183. [CrossRef]

28. Zhang, C.; Li, T.; Saker, S. Oscillation of fourth-order delay differential equations. J. Math. Sci. 2014, 201, 296-308. [CrossRef]

29. Bazighifan, O. On the oscillation of certain fourth-order differential equations with $p$-Laplacian like operator. Appl. Math. Comput. 2020, 386, 125475. [CrossRef]

30. Elabbasy, E.M.; Thandpani, E.; Moaaz, O.; Bazighifan, O. Oscillation of solutions to fourth-order delay differential equations with midlle term. Open J. Math. Sci. 2019, 3, 191-197. [CrossRef]

31. El-Deeb, A.A.-M.; Bazighifan, O.; Awrejcewicz, J. A Variety of Dynamic Steffensen-Type Inequalities on a General Time Scale. Symmetry 2021, 13, 1738. [CrossRef]

32. Chatzarakis, G.E.; Grace, S.R.; Jadlovska, I.; Li, T.; Tunç, E. Oscillation criteria for third-order Emden-Fowler differential equations with unbounded neutral coefficients. Complexity 2019, 2019, 5691758. [CrossRef]

33. Bazighifan, O.; Almutairi, A.; Almarri, B.; Marin, M. An Oscillation Criterion of Nonlinear Differential Equations with Advanced Term. Symmetry 2021, 13, 843. [CrossRef] 